\title{
Existence of rotating-periodic solutions for nonlinear second order vector differential equations
}

\author{
Jin Zhang ${ }^{1 *}$ and Xue Yang ${ }^{1,2}$
}

${ }^{*}$ Correspondence:

jinzhang@jlu.edu.cn

'School of Mathematics, Jilin University, Changchun, China Full list of author information is available at the end of the article

\begin{abstract}
In this paper, we establish two existence theorems of rotating-periodic solutions for nonlinear second order vector differential equations via the Leray-Schauder degree theory and the lower and upper solutions method. The concept "rotating-periodicity" is a kind of symmetry, which is a general version of periodicity, anti-periodicity, harmonic-periodicity, and it is also a special kind of quasi-periodicity. We also include several examples to illustrate the validity and applicability of our results.
\end{abstract}

MSC: Primary 34C25; 34B15; secondary 47H11

Keywords: Rotating-periodic solution; Leray-Schauder degree; Lower and upper solutions

\section{Introduction}

Periodicity is a very important property in the study of differential equations. Since Poincare established the existence of periodic solutions to the three-body problems, there has been a rich literature body on the periodic solutions of ordinary differential equations (see for example $[9,16,18]$ and the references therein). However, some differential equations often exhibit certain symmetries rather than periodicity, such as anti-periodicity, harmonic-periodicity, and quasi-periodicity. Recently, the existence of affine-periodic solutions and rotating-periodic solutions for nonlinear differential equations, which was firstly introduced in [24], has become a very interesting topic. Especially, Chang and Li $[4,5]$ studied the existence of rotating-periodic solutions for second order dynamical systems by using the coincidence degree theory. In [21, 23], the existence of affine-periodic solutions for nonlinear systems is obtained based on the existence of lower and upper solutions. One can also see [13-15] for the existence and multiplicity of rotating-periodic solutions of second order Hamiltonian systems.

This paper is devoted to investigating the existence of rotating-periodic solutions for the following second order vector differential equation:

$$
x^{\prime \prime}+f\left(t, x, x^{\prime}\right)=0,
$$

(c) The Author(s) 2020. This article is licensed under a Creative Commons Attribution 4.0 International License, which permits use, sharing, adaptation, distribution and reproduction in any medium or format, as long as you give appropriate credit to the original author(s) and the source, provide a link to the Creative Commons licence, and indicate if changes were made. The images or other third party material in this article are included in the article's Creative Commons licence, unless indicated otherwise in a credit line to the material. If material is not included in the article's Creative Commons licence and your intended use is not permitted by statutory regulation or exceeds the permitted use, you will need to obtain permission directly from the copyright holder. To view a copy of this licence, visit http://creativecommons.org/licenses/by/4.0/. 
where $f: \mathbb{R}^{1} \times \mathbb{R}^{n} \times \mathbb{R}^{n} \rightarrow \mathbb{R}^{n}$ is Lipschitz continuous. Assume, moreover, that there exist some $T>0$ and $Q \in O(n)$ such that

$$
f(t+T, x, y)=Q f\left(t, Q^{-1} x, Q^{-1} y\right), \quad \forall t \in \mathbb{R}^{1}, x, y \in \mathbb{R}^{n} .
$$

System (1)-(2) is called a $(Q, T)$-rotating-periodic system. A natural problem is seeking for a solution which can keep the rotating-symmetry of $f$, that is, the space variable $x$ has a rotation $Q$ as the time variable walks a period $T$, which leads to the following definition.

Definition 1 A function $x: \mathbb{R}^{1} \rightarrow \mathbb{R}^{n}$ is called a $(Q, T)$-rotating-periodic solution of system (1)-(2) if it is a solution of (1) on $\mathbb{R}^{1}$ and satisfies

$$
x(t+T)=Q x(t), \quad \forall t \in \mathbb{R}^{1} .
$$

There are some natural phenomena presenting rotating-periodicity, such as spiral waves which execute compound rotation (see $[3,20]$ for example), spiral line in geometry, or the orbit of the earth going around the sun.

Remark 1 The cases of $Q=I$ (the identical matrix) and $Q=-I$ correspond to the ones of $T$-periodic and $T$-anti-periodic solutions, respectively. If there exists some positive integer $N$ such that $Q^{N}=I$, then $(Q, T)$-rotating-periodic solutions are just harmonic solutions, i.e., $x(t+N T) \equiv x(t), \forall t \in \mathbb{R}^{1}$. In particular, when $Q=\operatorname{diag}\left(e^{i \theta_{1}}, \ldots, e^{i \theta_{n}}\right) \in O(n)$, our solutions are actually the usual quasi-periodic ones with the frequency $\left(\theta_{1}, \ldots, \theta_{n}\right)$.

In this paper, we shall investigate the existence of rotating-periodic solutions. The idea has largely been motivated by the work of Fabry and Habets [8] in which the existence of solutions was studied for the Picard boundary value problem. Topological methods, in particular the degree theory, some fixed point theorems, and lower and upper solutions method, are the most relevant tools in proving the existence of solutions for boundary value problems. Some remarkable works of this area can be found in $[1,2,6,7,11,12,19]$ and the references therein.

The rest of this paper is organized as follows: In Sect. 2, we mainly review some preliminary lemmas which will be needed for the proof of our existence theorems. In Sect. 3, we prove the first existence theorem of rotating-periodic solutions based on the LeraySchauder degree theory and a Bernstein-Nagumo type condition. In Sect. 4, by using the lower and upper solutions and a Kamke type condition, we establish another existence theorem of rotating-periodic solutions. To illustrate our results, Sect. 5 contains some examples which show the validity and applicability of the existence theorems.

\section{Preliminaries}

In this section, we present some preliminary lemmas in the form we need for the proof of the existence theorems.

The following lemma shows that the problem of finding rotating-periodic solutions can be reduced to the corresponding boundary value problem. 
Lemma $1 x(t)$ is a $(Q, T)$-rotating-periodic solution of system (1)-(2) if and only if $x(t)$ is a solution of $(1)$ and satisfies the following $(Q, T)$-rotating-periodic boundary conditions:

$$
x(T)=Q x(0), \quad x^{\prime}(T)=Q x^{\prime}(0) .
$$

In the following, we show that the boundary value problem (1)-(3) can be transformed into a fixed point problem.

In fact, if $I-Q \in \operatorname{GL}(n)$, integrating (1) twice over $[0, t]$ and taking into account the boundary conditions (3), we get the following integral equation:

$$
\begin{aligned}
x(t)= & (I-Q)^{-2} \int_{0}^{T}((t-s)(I-Q)-T Q) f\left(s, x(s), x^{\prime}(s)\right) \mathrm{d} s \\
& -\int_{0}^{t}(t-s) f\left(s, x(s), x^{\prime}(s)\right) \mathrm{d} s \\
\triangleq & \left(\mathcal{T}_{1} x\right)(t) .
\end{aligned}
$$

On the other hand, if $x(t)$ is a solution of (4), then differentiating (4) with respect to $t$ twice, we have $x^{\prime \prime}(t)=-f\left(t, x(t), x^{\prime}(t)\right)$. Substituting $t=0$ and $t=T$ into (4) and its derivative, we get $x(T)=Q x(0)$ and $x^{\prime}(T)=Q x^{\prime}(0)$.

If $I-Q \notin \mathrm{GL}(n)$, we can choose a constant $a>0$ such that $I-e^{a T} Q \in \mathrm{GL}(n)$ and $I-$ $e^{-a T} Q \in \mathrm{GL}(n)$ hold. Consider the following equation:

$$
x^{\prime \prime}-a^{2} x=-\left(a^{2} x+f\left(t, x, x^{\prime}\right)\right),
$$

which is equivalent to (1). From the theory of linear differential equations, we know that its solution $x(t)$ satisfies

$$
x(t)=c_{1} e^{a t}+c_{2} e^{-a t}+\frac{1}{2 a} \int_{0}^{t}\left(e^{-a(t-s)}-e^{a(t-s)}\right)\left(a^{2} x(s)+f\left(s, x(s), x^{\prime}(s)\right)\right) \mathrm{d} s .
$$

By the boundary conditions (3), we have

$$
\begin{aligned}
& c_{1}=\frac{1}{2 a}\left(I-e^{-a T} Q\right)^{-1} \int_{0}^{T} e^{-a s}\left(a^{2} x(s)+f\left(s, x(s), x^{\prime}(s)\right)\right) \mathrm{d} s, \\
& c_{2}=-\frac{1}{2 a}\left(I-e^{a T} Q\right)^{-1} \int_{0}^{T} e^{a s}\left(a^{2} x(s)+f\left(s, x(s), x^{\prime}(s)\right)\right) \mathrm{d} s .
\end{aligned}
$$

Then (1)-(3) is equivalent to the following integral equation:

$$
\begin{aligned}
x(t)= & \frac{1}{2 a}\left(I-e^{-a T} Q\right)^{-1} \int_{0}^{T} e^{a(t-s)}\left(a^{2} x(s)+f\left(s, x(s), x^{\prime}(s)\right)\right) \mathrm{d} s \\
& -\frac{1}{2 a}\left(I-e^{a T} Q\right)^{-1} \int_{0}^{T} e^{-a(t-s)}\left(a^{2} x(s)+f\left(s, x(s), x^{\prime}(s)\right)\right) \mathrm{d} s \\
& +\frac{1}{2 a} \int_{0}^{t}\left(e^{-a(t-s)}-e^{a(t-s)}\right)\left(a^{2} x(s)+f\left(s, x(s), x^{\prime}(s)\right)\right) \mathrm{d} s \\
\triangleq & \left(\mathcal{T}_{2} x\right)(t) .
\end{aligned}
$$


Hence, finding a solution of boundary value problem (1)-(3) is equivalent to finding a fixed point for operator $\mathcal{T}: C^{1}\left([0, T] ; \mathbb{R}^{n}\right) \rightarrow C^{1}\left([0, T] ; \mathbb{R}^{n}\right)$ defined by

$$
(\mathcal{T} x)(t)= \begin{cases}\left(\mathcal{T}_{1} x\right)(t) & \text { if } I-Q \in \mathrm{GL}(n), \\ \left(\mathcal{T}_{2} x\right)(t) & \text { if } I-Q \notin \mathrm{GL}(n),\end{cases}
$$

where $C^{1}\left([0, T] ; \mathbb{R}^{n}\right)$ is the Banach space of $C^{1}$ functions $x:[0, T] \rightarrow \mathbb{R}^{n}$ with the norm

$$
\|x\|_{1}=\max \left\{\max _{t \in[0, T]}|x(t)|, \max _{t \in[0, T]}\left|x^{\prime}(t)\right|\right\} .
$$

Throughout the paper, it is convenient to employ $|x|$ as the notation for the Euclidean norm in $\mathbb{R}^{n}$ and $\langle x, y\rangle$ for the scalar product of two vectors $x, y \in \mathbb{R}^{n}$.

The existence of a fixed point for operator $\mathcal{T}$ will be based on the following lemma given in [8].

Lemma 2 Let $X$ be a Banach space, $\mathcal{A}: X \rightarrow X$ be a compact operator such that $\mathcal{A}$ has a unique fixed point $x_{0}$, and $\Omega$ be an open bounded set such that $x_{0} \in \Omega$. Then the compact operator $\mathcal{T}: \bar{\Omega} \rightarrow$ X has a fixed point in $\bar{\Omega}$ if, for any $\lambda \in(0,1)$, the following equation

$$
x=\lambda \mathcal{T} x+(1-\lambda) \mathcal{A} x
$$

has no solution $x$ on the boundary $\partial \Omega$ of $\Omega$.

In this paper, we use the following Bernstein-Nagumo type lemma (see [8]), which is an immediate application of results given by Mawhin [17], from which a priori bound for the possible solutions can be obtained.

Lemma 3 Let $x:[0, T] \rightarrow \mathbb{R}^{n}$ be an absolutely continuous function with an absolutely continuous derivative. Assume that, for almost every $t \in[0, T]$, we have $\left|\left\langle x^{\prime}(t), x^{\prime \prime}(t)\right\rangle\right| \leq$ $h\left(\left|x^{\prime}(t)\right|\right)\left|x^{\prime}(t)\right|$, where $h: \mathbb{R}^{+} \rightarrow \mathbb{R}^{+} \backslash\{0\}$ is continuous and satisfies

$$
\int_{0}^{+\infty} \frac{s^{2} \mathrm{~d} s}{h(s)}=+\infty
$$

Then $\left|x^{\prime}(t)\right| \leq g\left(\int_{0}^{T}\left|x^{\prime}(t)\right|^{2} \mathrm{~d} t\right)$, where $g$ is defined by

$$
\int_{\sqrt{u / T}}^{g(u)} \frac{s^{2}}{h(s)} \mathrm{d} s=u
$$

\section{The existence theorem via Leray-Schauder degree theory}

In this section, we prove the existence theorem about rotating-periodic solutions by means of the Leray-Schauder degree theory. As it is pointed out in Sect. 2, we only need to prove the existence of fixed points of operator $\mathcal{T}$ defined by (5).

Since $f: \mathbb{R}^{1} \times \mathbb{R}^{n} \times \mathbb{R}^{n} \rightarrow \mathbb{R}^{n}$ is continuous, the operator $\mathcal{T}$ is compact. We want to show that under appropriate conditions on $f$, one can build a suitable operator $\mathcal{A}$ and a 
set $\Omega$ such that Lemma 2 can be applied. To this purpose, in the following theorem, let the compact operator $\mathcal{A}: C^{1}\left([0, T] ; \mathbb{R}^{n}\right) \rightarrow C^{1}\left([0, T] ; \mathbb{R}^{n}\right)$ be defined as follows:

$$
(\mathcal{A} x)(t)= \begin{cases}\left(\mathcal{A}_{1} x\right)(t) & \text { if } I-Q \in \mathrm{GL}(n), \\ \left(\mathcal{A}_{2} x\right)(t) & \text { if } I-Q \notin \mathrm{GL}(n),\end{cases}
$$

where

$$
\begin{aligned}
\left(\mathcal{A}_{1} x\right)(t)= & (I-Q)^{-2} \int_{0}^{T}(T Q-(t-s)(I-Q)) k^{2} x(s) \mathrm{d} s+\int_{0}^{t}(t-s) k^{2} x(s) \mathrm{d} s, \\
\left(\mathcal{A}_{2} x\right)(t)= & \frac{1}{2 a}\left(I-e^{-a T} Q\right)^{-1} \int_{0}^{T} e^{a(t-s)}\left(a^{2}-k^{2}\right) x(s) \mathrm{d} s \\
& -\frac{1}{2 a}\left(I-e^{a T} Q\right)^{-1} \int_{0}^{T} e^{-a(t-s)}\left(a^{2}-k^{2}\right) x(s) \mathrm{d} s \\
& +\frac{1}{2 a} \int_{0}^{t}\left(e^{-a(t-s)}-e^{a(t-s)}\right)\left(a^{2}-k^{2}\right) x(s) \mathrm{d} s
\end{aligned}
$$

for $k>0$, and let the open bounded set $\Omega$ be defined as follows:

$$
\Omega=\left\{x \in C^{1}\left([0, T] ; \mathbb{R}^{n}\right)|| x(t)|<\phi(t),| x^{\prime}(t) \mid<\rho, \forall t \in[0, T]\right\},
$$

where $\phi:[0, T] \rightarrow \mathbb{R}^{+} \backslash\{0\}$ is some strictly positive $C^{2}$ function and $\rho>0$. Then we have the following existence result.

Theorem 1 Suppose that there exists a $C^{2}$ function $\phi:[0, T] \rightarrow \mathbb{R}^{+} \backslash\{0\}$ with $\phi(0)=\phi(T)$, $\phi^{\prime}(0)=\phi^{\prime}(T)$ such that, for any $t \in[0, T],|x|=\phi(t)$, and $\langle x, y\rangle=|x| \phi^{\prime}(t)$, we have

$$
\langle x, f(t, x, y)\rangle \leq|y|^{2}-\phi(t) \phi^{\prime \prime}(t)-\phi^{\prime}(t)^{2} .
$$

Assume, moreover, that there exist constants $\alpha, \beta \geq 0,0 \leq \gamma<1$, and $\delta>0$ such that, for all $t \in[0, T],|x| \leq \phi(t)$, and $y \in \mathbb{R}^{n}$, we have

$$
\begin{aligned}
& \langle x, f(t, x, y)\rangle \leq \alpha|x|^{2}+\beta|x||y|+\gamma|y|^{2}+\delta, \\
& |\langle y, f(t, x, y)\rangle| \leq h_{0}(|y|)|y|
\end{aligned}
$$

where $h_{0}: \mathbb{R}^{+} \rightarrow \mathbb{R}^{+} \backslash\{0\}$ is increasing, continuous and satisfies (7).

Then the operator $\mathcal{T}$ has at least one fixed point $x^{*}$ such that $\left|x^{*}(t)\right| \leq \phi(t), \forall t \in[0, T]$, that is, $(Q, T)$-rotating-periodic system (1)-(2) has at least one $(Q, T)$-rotating-periodic solution $x^{*}(t)$.

Proof Let $\mathcal{A}$ be defined by (8) and $\Omega$ by (9). We suppose that $\mathcal{T}$ defined by (5) has no fixed point on $\partial \Omega$; otherwise, Theorem 1 has been proven.

According to Lemma 2, we firstly prove that $\mathcal{A}$ has a unique fixed point in $\Omega$. By similar arguments in Sect. 2, we know that $\mathcal{A} x=x$ is equivalent to the following boundary value 
problem:

$$
\begin{aligned}
& x^{\prime \prime}=k^{2} x, \\
& x(T)=Q x(0), \quad x^{\prime}(T)=Q x^{\prime}(0) .
\end{aligned}
$$

The solution of (13) can be explicitly written as $x(t)=h_{1} e^{k t}+h_{2} e^{-k t}$, where $h_{1}$ and $h_{2}$ satisfy $\left(Q-e^{k T} I\right) h_{1}=0,\left(Q-e^{-k T} I\right) h_{2}=0$. We can choose sufficiently large $k$ such that $e^{k T}$ and $e^{-k T}$ are not the eigenvalues of $Q$, then $h_{1}=h_{2}=0$. It follows that the unique solution of (13) is $x_{0}=0$, and thus operator $\mathcal{A}$ has a unique fixed point $x_{0} \in \Omega$.

Secondly, it suffices to show that if $\rho$ and $k$ are chosen large enough, there is no solution of (6) on $\partial \Omega$ for $\lambda \in(0,1)$. Consider the following homotopy:

$$
\mathcal{H}(x, \lambda)=: \lambda \mathcal{T} x+(1-\lambda) \mathcal{A} x, \quad(x, \lambda) \in \bar{\Omega} \times[0,1] .
$$

Since there is no fixed point of $\mathcal{H}(\cdot, 1)=\mathcal{T}$ on $\partial \Omega$, it follows from the compactness of $\mathcal{H}$ that there exists $\lambda_{0} \in(0,1)$ such that the operator $\mathcal{H}(\cdot, \lambda)$ has no fixed point on $\partial \Omega$ for $\lambda \in\left[\lambda_{0}, 1\right]$. Thus, it suffices to show that there is no solution of (6) on $\partial \Omega$ for $\lambda \in\left(0, \lambda_{0}\right)$.

If, on the other hand, there exists a solution $x \in \partial \Omega$ of (6), then the following situations hold: either there exists $\xi \in[0, T]$ such that $|x(t)|^{2}-\phi^{2}(t)$ reaches the maximum value 0 at $t=\xi$, or there exists $\eta \in[0, T]$ such that $\left|x^{\prime}(\eta)\right|=\rho$. In what follows we will prove that none of these two statements hold for sufficiently large $\rho$.

Assume that $|x(t)|^{2}-\phi^{2}(t)$ reaches the maximum value 0 at $t=\xi \in[0, T]$. If $\xi \in(0, T)$, then we have

$$
|x(\xi)|=\phi(\xi), \quad\left\langle x(\xi), x^{\prime}(\xi)\right\rangle-\phi(\xi) \phi^{\prime}(\xi)=0
$$

Hence

$$
\left\langle x(\xi), x^{\prime \prime}(\xi)\right\rangle+\left|x^{\prime}(\xi)\right|^{2}-\phi(\xi) \phi^{\prime \prime}(\xi)-\phi^{\prime}(\xi)^{2} \leq 0
$$

Since $x$ is a solution of (6), it follows that

$$
x^{\prime \prime}(t)=-\lambda f\left(t, x(t), x^{\prime}(t)\right)+(1-\lambda) k^{2} x(t)
$$

Using this equation together with (10), we have that

$$
\begin{aligned}
& \left\langle x(\xi), x^{\prime \prime}(\xi)\right\rangle+\left|x^{\prime}(\xi)\right|^{2}-\phi(\xi) \phi^{\prime \prime}(\xi)-\phi^{\prime}(\xi)^{2} \\
& \quad=-\lambda\left\langle x(\xi), f\left(\xi, x(\xi), x^{\prime}(\xi)\right)\right\rangle+(1-\lambda) k^{2}|x(\xi)|^{2}+\left|x^{\prime}(\xi)\right|^{2}-\phi(\xi) \phi^{\prime \prime}(\xi)-\phi^{\prime}(\xi)^{2} \\
& \quad \geq(1-\lambda)\left(k^{2} \phi(\xi)^{2}+\left|x^{\prime}(\xi)\right|^{2}-\phi(\xi) \phi^{\prime \prime}(\xi)-\phi^{\prime}(\xi)^{2}\right)>0
\end{aligned}
$$

holds for all $\lambda \in\left(0, \lambda_{0}\right)$ and sufficiently large $k$. This contradicts (14). If $\xi=0$, that is, $|x(t)|^{2}-\phi^{2}(t)$ reaches the maximum value 0 at $t=0$, then from (3) and the orthogonality of $Q$, we have

$$
|x(T)|^{2}-\phi^{2}(T)=|x(0)|^{2}-\phi^{2}(0)=0,
$$


which implies that $|x(t)|^{2}-\phi^{2}(t)$ reaches the maximum value 0 at $t=T$, and vice versa. Hence,

$$
\left\langle x(0), x^{\prime}(0)\right\rangle-\phi(0) \phi^{\prime}(0) \leq 0, \quad\left\langle x(T), x^{\prime}(T)\right\rangle-\phi(T) \phi^{\prime}(T) \geq 0 .
$$

From (3), the orthogonality of $Q$, and the conditions of $\phi$, we conclude that

$$
\left\langle x(T), x^{\prime}(T)\right\rangle-\phi(T) \phi^{\prime}(T)=\left\langle x(0), x^{\prime}(0)\right\rangle-\phi(0) \phi^{\prime}(0)=0 .
$$

Then it follows that

$$
\begin{aligned}
& \left\langle x(0), x^{\prime \prime}(0)\right\rangle+\left|x^{\prime}(0)\right|^{2}-\phi(0) \phi^{\prime \prime}(0)-\phi^{\prime}(0)^{2} \leq 0, \\
& \left\langle x(T), x^{\prime \prime}(T)\right\rangle+\left|x^{\prime}(T)\right|^{2}-\phi(T) \phi^{\prime \prime}(T)-\phi^{\prime}(T)^{2} \leq 0 .
\end{aligned}
$$

Hence, we can also deduce a contradiction.

Next, we will prove that, for any solution $x$ of (6) satisfying the condition $|x(t)| \leq \phi(t)$, $\left|x^{\prime}(t)\right|$ is necessarily bounded, regardless of the choice of $\lambda \in\left(0, \lambda_{0}\right)$. Integrating the following equality over $[0, T]$

$$
\left\langle x(t), x^{\prime \prime}(t)\right\rangle=-\lambda\left\langle x(t), f\left(t, x(t), x^{\prime}(t)\right)\right\rangle+(1-\lambda) k^{2}|x(t)|^{2}
$$

and taking into account (3) and the orthogonality of $Q$, we have

$$
\int_{0}^{T}\left|x^{\prime}(t)\right|^{2} \mathrm{~d} t=\lambda \int_{0}^{T}\left\langle x(t), f\left(t, x(t), x^{\prime}(t)\right)\right\rangle \mathrm{d} t-(1-\lambda) k^{2} \int_{0}^{T}|x(t)|^{2} \mathrm{~d} t .
$$

It follows from (11) that, if $|x(t)| \leq \phi(t)$, then

$$
\begin{aligned}
& \int_{0}^{T}\left|x^{\prime}(t)\right|^{2} \mathrm{~d} t \\
& \leq \lambda\left(\alpha \int_{0}^{T}|x(t)|^{2} \mathrm{~d} t+\beta \int_{0}^{T}|x(t)|\left|x^{\prime}(t)\right| \mathrm{d} t+\gamma \int_{0}^{T}\left|x^{\prime}(t)\right|^{2} \mathrm{~d} t+\delta T\right) \\
& \quad-(1-\lambda) k^{2} \int_{0}^{T}|x(t)|^{2} \mathrm{~d} t \\
& \leq \\
& \leq {\left[\lambda(\alpha+\beta / 2 \epsilon)-(1-\lambda) k^{2}\right] \int_{0}^{T}|x(t)|^{2} \mathrm{~d} t+\lambda(\gamma+\epsilon \beta / 2) \int_{0}^{T}\left|x^{\prime}(t)\right|^{2} \mathrm{~d} t+\lambda \delta T, }
\end{aligned}
$$

where $\epsilon$ is a positive number provided $\gamma+\epsilon \beta / 2<1$. Take $k$ large enough such that $(1-$ $\left.\lambda_{0}\right) k^{2} \geq \alpha+\beta / 2 \epsilon$, then for all $\lambda \in\left(0, \lambda_{0}\right)$, we have

$$
\int_{0}^{T}\left|x^{\prime}(t)\right|^{2} \mathrm{~d} t \leq \frac{\delta T}{1-(\gamma+\epsilon \beta / 2)} \triangleq K .
$$

Using (12), we have

$$
\begin{aligned}
\left|\left\langle x^{\prime}(t), x^{\prime \prime}(t)\right\rangle\right| & \left.\leq \lambda|| x^{\prime}(t), f\left(t, x(t), x^{\prime}(t)\right)\right\rangle\left|+(1-\lambda) k^{2}\right| x(t)|| x^{\prime}(t) \mid \\
& \leq \lambda h_{0}\left(\left|x^{\prime}(t)\right|\right)\left|x^{\prime}(t)\right|+(1-\lambda) k^{2} M\left|x^{\prime}(t)\right| \\
& \leq\left(h_{0}\left(\left|x^{\prime}(t)\right|\right)+k^{2} M\right)\left|x^{\prime}(t)\right| \triangleq h\left(\left|x^{\prime}(t)\right|\right)\left|x^{\prime}(t)\right|,
\end{aligned}
$$


where $M$ is an upper bound of $\phi(t)$ on $[0, T]$. Since $h_{0}$ is increasing and satisfies (7), it is true for $h$ as well. Then it follows from Lemma 3 that an upper bound for $\left|x^{\prime}(t)\right|$ is found:

$$
\left|x^{\prime}(t)\right| \leq g\left(\int_{0}^{T}\left|x^{\prime}(t)\right|^{2} \mathrm{~d} t\right) \leq g(K), \quad \forall t \in[0, T]
$$

Hence, we can take $\rho>g(K)$ in the definition of $\Omega$ in (9) such that there is no solution of (6) on $\partial \Omega$ for $\lambda \in(0,1)$. Thereby the existence of a solution $x^{*} \in \bar{\Omega}$ is easily obtained from Lemma 2.

\section{The existence theorem via lower and upper solutions}

Using the lower and upper solutions method, we shall establish another existence theorem about rotating-periodic solutions. The main idea is that if we can find a lower solution which is smaller than an upper one, there is a solution wedged between these two.

To obtain the existence result for vector differential equations, we need the partial orders in $\mathbb{R}^{n}$. The positive cone in $\mathbb{R}^{n}$ is the set of all $n$ tuples with nonnegative coordinates, which gives rise to a partial order on $\mathbb{R}^{n}$ in the following way:

$$
\begin{aligned}
& x \leq y \quad \Leftrightarrow \quad x_{i} \leq y_{i} \quad \text { for } i=1, \ldots, n, \\
& x<y \quad \Leftrightarrow \quad x_{i}<y_{i} \quad \text { for } i=1, \ldots, n,
\end{aligned}
$$

where $x, y \in \mathbb{R}^{n}, x_{i}$ and $y_{i}$ are the $i$ th components of $x$ and $y$, respectively.

Before proceeding to the existence theorem, we also need to introduce the following definition of lower and upper solutions.

Definition 2 The $C^{2}$ functions $\varphi$ and $\psi: \mathbb{R}^{1} \rightarrow \mathbb{R}^{n}$ are said to be lower and upper solutions of (1), respectively, if for all $t \in \mathbb{R}^{1}, \varphi(t) \leq \psi(t)$ and

$$
\varphi^{\prime \prime}(t)+f\left(t, \varphi(t), \varphi^{\prime}(t)\right) \geq 0, \quad \psi^{\prime \prime}(t)+f\left(t, \psi(t), \psi^{\prime}(t)\right) \leq 0
$$

Furthermore, the following Kamke type condition is needed in the proof of the existence theorem, whose general form can be found in [10].

(K) For any $t \in \mathbb{R}^{1}$ and $i=1, \ldots, n$,

$$
\varphi_{i}^{\prime \prime}(t)+f_{i}(t, \hat{x}(t), \hat{y}(t)) \geq 0, \quad \psi_{i}^{\prime \prime}(t)+f_{i}(t, \tilde{x}(t), \tilde{y}(t)) \leq 0,
$$

where $\varphi(t) \leq \hat{x}(t), \tilde{x}(t) \leq \psi(t)$ with the $i$ th components of $\hat{x}(t), \tilde{x}(t), \hat{y}(t)$, and $\tilde{y}(t)$ are $\varphi_{i}(t)$, $\psi_{i}(t), \varphi_{i}^{\prime}(t)$, and $\psi_{i}^{\prime}(t)$, respectively.

Now we are in a position to state the following existence result about rotating-periodic solutions for system (1)-(2).

Theorem 2 Suppose that the following conditions hold:

(i) There exist $C^{2}$ lower and upper solutions $\varphi(t)<\psi(t)$ of (1) which are T-periodic and satisfy the Kamke type condition (K);

(ii) There exist some $c>0$ and a $C^{2}$ function $\sigma: \mathbb{R}^{1} \rightarrow \mathbb{R}^{n}$ which is $(Q, T)$-rotating-periodic such that

$$
\varphi(t) \leq \sigma(t)-(c, \ldots, c)^{T}<\sigma(t)<\sigma(t)+(c, \ldots, c)^{T} \leq \psi(t), \quad \forall t \in \mathbb{R}^{1} ;
$$


(iii) There exist constants $\alpha, \beta \geq 0,0 \leq \gamma<1$, and $\delta>0$ such that, for any $t \in \mathbb{R}^{1}$, $\varphi(t) \leq x \leq \psi(t)$, and $y \in \mathbb{R}^{n}$, we have

$$
\begin{aligned}
& \langle x, f(t, x, y)\rangle \leq \alpha|x|^{2}+\beta|x||y|+\gamma|y|^{2}+\delta \\
& |\langle y, f(t, x, y)\rangle| \leq h_{0}(|y|)|y|
\end{aligned}
$$

where $h_{0}: \mathbb{R}^{+} \rightarrow \mathbb{R}^{+} \backslash\{0\}$ is increasing, continuous and satisfies (7).

Then $(Q, T)$-rotating-periodic system (1)-(2) has at least one $(Q, T)$-rotating-periodic solution $x^{*}(t)$ satisfying $\varphi(t) \leq x^{*}(t) \leq \psi(t), \forall t \in \mathbb{R}^{1}$.

Proof As in Theorem 1, the result follows immediately if one applies Lemma 2 to a suitable operator $\mathcal{A}$ and an open set $\Omega$. Let $X$ be the Banach space defined by

$$
X=\left\{x \in C^{1}\left(\mathbb{R}^{1} ; \mathbb{R}^{n}\right) \mid x(t+T)=Q x(t), \forall t \in \mathbb{R}^{1}\right\}
$$

with the norm $\|\cdot\|_{1}$. Then, from the orthogonality of $Q$, we have that

$$
\Omega=\left\{x \in X|\varphi(t)<x(t)<\psi(t),| x^{\prime}(t) \mid<\rho, \forall t \in \mathbb{R}^{1}\right\}
$$

is a nonempty open and bounded set in $X$. Define

$$
(\mathcal{A} x)(t)= \begin{cases}\left(\mathcal{A}_{1} x\right)(t) & \text { if } I-Q \in \mathrm{GL}(n), \\ \left(\mathcal{A}_{2} x\right)(t) & \text { if } I-Q \notin \mathrm{GL}(n),\end{cases}
$$

where

$$
\begin{aligned}
\left(\mathcal{A}_{1} x\right)(t)= & (I-Q)^{-2} \int_{0}^{T}(T Q-(t-s)(I-Q))\left(k^{2} x(s)+\sigma^{\prime \prime}(s)-k^{2} \sigma(s)\right) \mathrm{d} s \\
& +\int_{0}^{t}(t-s)\left(k^{2} x(s)+\sigma^{\prime \prime}(s)-k^{2} \sigma(s)\right) \mathrm{d} s, \\
\left(\mathcal{A}_{2} x\right)(t)= & \frac{1}{2 a}\left(I-e^{-a T} Q\right)^{-1} \int_{0}^{T} e^{a(t-s)}\left(\left(a^{2}-k^{2}\right) x(s)-\sigma^{\prime \prime}(s)+k^{2} \sigma(s)\right) \mathrm{d} s \\
& -\frac{1}{2 a}\left(I-e^{a T} Q\right)^{-1} \int_{0}^{T} e^{-a(t-s)}\left(\left(a^{2}-k^{2}\right) x(s)-\sigma^{\prime \prime}(s)+k^{2} \sigma(s)\right) \mathrm{d} s \\
& +\frac{1}{2 a} \int_{0}^{t}\left(e^{-a(t-s)}-e^{a(t-s)}\right)\left(\left(a^{2}-k^{2}\right) x(s)-\sigma^{\prime \prime}(s)+k^{2} \sigma(s)\right) \mathrm{d} s .
\end{aligned}
$$

Then the operator $\mathcal{T}$ defined by (5) and the operator $\mathcal{A}$ defined by (19) are both compact operators from $X$ to $X$. As in Sect. 2, we know that $\mathcal{A} x=x$ is equivalent to the following boundary value problem:

$$
\begin{aligned}
& x^{\prime \prime}-k^{2} x=\sigma^{\prime \prime}-k^{2} \sigma, \\
& x(T)=Q x(0), \quad x^{\prime}(T)=Q x^{\prime}(0) .
\end{aligned}
$$

Similarly, we can prove that the unique solution of $(20)$ is $x_{0}(t)=\sigma(t)$ for sufficiently large $k$. It means that $x_{0}$ is the unique fixed point of $\mathcal{A}$ and $x_{0} \in \Omega$. 
Furthermore, it suffices to show that there is no solution of (6) on $\partial \Omega$ for $\lambda \in\left(0, \lambda_{0}\right)$ with $\lambda_{0} \in(0,1)$. Let $x \in \partial \Omega$ be a solution of (6). Then $\varphi(t) \leq x(t) \leq \psi(t)$ for all $t \in \mathbb{R}^{1}$, and either there exist $\xi \in \mathbb{R}^{1}$ and $i \in\{1, \ldots, n\}$ such that $x_{i}(t)-\psi_{i}(t)$ (or $\varphi_{i}(t)-x_{i}(t)$ ) reaches the maximum value 0 at $t=\xi$, or there exists $\eta \in \mathbb{R}^{1}$ such that $\left|x^{\prime}(\eta)\right|=\rho$.

Without loss of generality, assume that $x_{i}(t)-\psi_{i}(t)$ reaches the maximum value 0 at $t=\xi \in \mathbb{R}^{1}$. Then the following relations hold:

$$
x_{i}(\xi)=\psi_{i}(\xi), \quad x_{i}^{\prime}(\xi)=\psi_{i}^{\prime}(\xi), \quad x_{i}^{\prime \prime}(\xi) \leq \psi_{i}^{\prime \prime}(\xi) .
$$

Since $x$ is a solution of (6), we have

$$
x_{i}^{\prime \prime}(t)=-\lambda f_{i}\left(t, x(t), x^{\prime}(t)\right)+(1-\lambda)\left(k^{2} x_{i}(t)+\sigma_{i}^{\prime \prime}(t)-k^{2} \sigma_{i}(t)\right) .
$$

Taking $k$ large enough such that $c k^{2}>\max _{t \in \mathbb{R}^{1}}\left|\psi^{\prime \prime}(t)-\sigma^{\prime \prime}(t)\right|$, by (15) and (16), we have

$$
\begin{aligned}
x_{i}^{\prime \prime}(\xi) & =-\lambda f_{i}\left(\xi, x(\xi), x^{\prime}(\xi)\right)+(1-\lambda)\left(k^{2} \psi_{i}(\xi)+\sigma_{i}^{\prime \prime}(\xi)-k^{2} \sigma_{i}(\xi)\right) \\
& >\lambda \psi_{i}^{\prime \prime}(\xi)+(1-\lambda) \psi_{i}^{\prime \prime}(\xi)=\psi_{i}^{\prime \prime}(\xi),
\end{aligned}
$$

which is a contradiction.

Next, we will show that, for any solution $x$ of (6) with the condition $\varphi(t) \leq x(t) \leq \psi(t)$, there is a certain number $K>0$ such that the estimate $\left|x^{\prime}(t)\right|<K$ holds, regardless of the choice of $\lambda \in\left(0, \lambda_{0}\right)$. Since $x$ is a solution of (6), we have

$$
\left\langle x(t), x^{\prime \prime}(t)\right\rangle=-\lambda\left\langle x(t), f\left(t, x(t), x^{\prime}(t)\right)\right\rangle+(1-\lambda)\left\langle x(t), k^{2} x(t)+\sigma^{\prime \prime}(t)-k^{2} \sigma(t)\right\rangle .
$$

It follows from (3) and (17) that, if $\varphi(t) \leq x(t) \leq \psi(t)$,

$$
\begin{aligned}
& \int_{0}^{T}\left|x^{\prime}(t)\right|^{2} \mathrm{~d} t \\
& =\lambda \int_{0}^{T}\left\langle x(t), f\left(t, x(t), x^{\prime}(t)\right)\right\rangle \mathrm{d} t-(1-\lambda) \int_{0}^{T}\left\langle x(t), k^{2} x(t)+\sigma^{\prime \prime}(t)-k^{2} \sigma(t)\right\rangle \mathrm{d} t \\
& \quad \leq\left[\lambda(\alpha+\beta / 2 \epsilon)-(1-\lambda) k^{2}\right] \int_{0}^{T}|x(t)|^{2} \mathrm{~d} t+\lambda(\gamma+\epsilon \beta / 2) \int_{0}^{T}\left|x^{\prime}(t)\right|^{2} \mathrm{~d} t \\
& \quad+\lambda \delta T+(1-\lambda) \int_{0}^{T}\left\langle x(t), k^{2} \sigma(t)-\sigma^{\prime \prime}(t)\right\rangle \mathrm{d} t,
\end{aligned}
$$

where $\epsilon$ is a positive number provided $\gamma+\epsilon \beta / 2<1$. Then, for sufficiently large $k$ and all $\lambda \in\left(0, \lambda_{0}\right)$, we have

$$
\int_{0}^{T}\left|x^{\prime}(t)\right|^{2} \mathrm{~d} t \leq \frac{(\delta+M) T}{1-(\gamma+\epsilon \beta / 2)}
$$

where $M$ is an upper bound of $\left|\left\langle\varphi(t), k^{2} \sigma(t)-\sigma^{\prime \prime}(t)\right\rangle\right|$ and $\left|\left\langle\psi(t), k^{2} \sigma(t)-\sigma^{\prime \prime}(t)\right\rangle\right|$ on $[0, T]$. Then, using Lemma 3, an upper bound for $\left|x^{\prime}(t)\right|$ on $[0, T]$ can be found. Furthermore, using the rotating-periodicity of $x$ and the orthogonality of $Q$, we can obtain an upper bound for $\left|x^{\prime}(t)\right|$ on $\mathbb{R}^{1}$. This completes the proof. 


\section{Some applications}

In this section, we apply the techniques described in the preceding sections to study the existence of rotating-periodic solutions for some special types of equations under the corresponding rotating-periodic conditions. We need the following partial orders in $\mathbb{R}^{n \times n}$ :

$$
\begin{aligned}
& A \leq B \quad \Leftrightarrow \quad a_{i j} \leq b_{i j} \quad \text { for } i, j=1, \ldots, n, \\
& A<B \quad \Leftrightarrow \quad a_{i j}<b_{i j} \quad \text { for } i, j=1, \ldots, n,
\end{aligned}
$$

where $A, B \in \mathbb{R}^{n \times n}, a_{i j}$ and $b_{i j}$ are the $(i, j)$ th components of $A$ and $B$, respectively. Assume, moreover, that the norm is denoted by $\|A\|$ for $A \in \mathbb{R}^{n \times n}$.

Example 1 Consider the following second order equation:

$$
x^{\prime \prime}-a(t)|x|^{2 v} x=e(t)
$$

where $v>0$ is a constant, $a: \mathbb{R}^{1} \rightarrow \mathbb{R}^{+} \backslash\{0\}$ and $e: \mathbb{R}^{1} \rightarrow \mathbb{R}^{n}$ are continuous functions, and there exist some $T>0$ and $Q \in O(n)$ such that

$$
a(t+T)=a(t), \quad e(t+T)=Q e(t), \quad \forall t \in \mathbb{R}^{1} .
$$

Then $(Q, T)$-rotating-periodic system (21)-(22) has at least one $(Q, T)$-rotating-periodic solution.

Proof Let $\phi(t)=C$, where $C>0$ is a constant. Then $\phi$ is a strictly positive $C^{2}$ function with $\phi(0)=\phi(T), \phi^{\prime}(0)=\phi^{\prime}(T)$. Moreover, for any $t \in[0, T],|x|=C$, we have

$$
\left\langle x,-a(t)|x|^{2 v} x-e(t)\right\rangle=-a(t)|x|^{2(v+1)}-\langle x, e(t)\rangle \leq 0
$$

for $C \geq \max \left\{1, M_{e} / m_{a}\right\}$, where $m_{a}=\min _{t \in[0, T]} a(t), M_{e}=\max _{t \in[0, T]}|e(t)|$. Furthermore, for any $t \in[0, T],|x| \leq C$, and $y \in \mathbb{R}^{n}$, we have

$$
\left\langle x,-a(t)|x|^{2 v} x-e(t)\right\rangle \leq M_{e} C, \quad\left|\left\langle y,-a(t)|x|^{2 v} x-e(t)\right\rangle\right| \leq\left(M_{a} C^{2 v+1}+M_{e}\right)|y|,
$$

where $M_{a}=\max _{t \in[0, T]} a(t)$. Denote $h_{0}(s)=M_{a} C^{2 v+1}+M_{e}$, which is increasing, continuous and satisfies (7). This means that conditions (10), (11), and (12) in Theorem 1 hold. Then there exists a $(Q, T)$-rotating-periodic solution.

Example 2 Consider the following second order equation:

$$
x^{\prime \prime}+f(t, x)=0
$$

where $f: \mathbb{R}^{1} \times \mathbb{R}^{n} \rightarrow \mathbb{R}^{n}$ is a $C^{1}$ function. Equation (23) describes the motion of some interesting problems in applications such as the Brillouin focusing system, where the equation is a singular perturbation of the Mathieu equation [22]. The so-called Ermakov-Pinney equation and Hill equation are special cases of (23). 
Suppose that there exist some $T>0$ and $Q \in O(n)$ such that

$$
f(t+T, x)=Q f\left(t, Q^{-1} x\right), \quad \forall t \in \mathbb{R}^{1}, x \in \mathbb{R}^{n},
$$

and the following condition holds:

$$
f_{x}(t, x) \leq-\kappa I, \quad \forall t \in \mathbb{R}^{1}, x \in \mathbb{R}^{n}
$$

where $f_{x}$ is the Hessian matrix of $f, \kappa>0$ is a constant. Then $(Q, T)$-rotating-periodic system (23)-(24) has at least one $(Q, T)$-rotating-periodic solution.

Proof Let $\phi(t)=C$ for some $C>0$. For any $t \in[0, T],|x|=C$, we have

$$
\langle x, f(t, x)\rangle=\left\langle x, f(t, 0)+f_{x}(t, \xi(t)) x\right\rangle \leq\langle x, f(t, 0)\rangle-\kappa|x|^{2} \leq 0
$$

for $C \geq M_{0} / \kappa$, where $M_{0}=\max _{t \in[0, T]}|f(t, 0)|$. Furthermore, for any $t \in[0, T],|x| \leq C$, and $y \in \mathbb{R}^{n}$, we have

$$
\langle x, f(t, x)\rangle \leq-\kappa|x|^{2}+M_{0} C, \quad|\langle y, f(t, x)\rangle| \leq M_{f}|y|
$$

where $M_{f}$ is an upper bound of $|f(t, x)|$ for $t \in[0, T]$ and $|x| \leq C$. Then, according to Theorem 1 , there exists a $(Q, T)$-rotating-periodic solution.

Example 3 Consider the following second order equation:

$$
x^{\prime \prime}+A(t, x) x^{\prime}+B(t) x=e(t)
$$

where $A=\operatorname{diag}\left(a_{11}, \ldots, a_{n n}\right): \mathbb{R}^{1} \times \mathbb{R}^{n} \rightarrow \mathbb{R}^{n \times n}$ is a $C^{1}$ function, $B=\left(b_{i j}\right)_{n \times n}: \mathbb{R}^{1} \rightarrow \mathbb{R}^{n \times n}$ and $e: \mathbb{R}^{1} \rightarrow \mathbb{R}^{n}$ are continuous functions.

Suppose that there exist some $T>0$ and $Q \in O(n)$ such that

$$
\begin{aligned}
& A(t+T, x)=Q A\left(t, Q^{-1} x\right) Q^{-1}, \quad \forall t \in \mathbb{R}^{1}, x \in \mathbb{R}^{n}, \\
& B(t+T)=Q B(t) Q^{-1}, \quad e(t+T)=Q e(t), \quad \forall t \in \mathbb{R}^{1},
\end{aligned}
$$

and the following condition holds: for all $i=1, \ldots, n$,

$$
-b_{i i}(t)>\sum_{j \neq i}\left|b_{i j}(t)\right|+\left|e_{i}(t)\right|, \quad \forall t \in \mathbb{R}^{1} .
$$

Then $(Q, T)$-rotating-periodic system (25)-(26) has at least one $(Q, T)$-rotating-periodic solution.

Proof Let $\sigma: \mathbb{R}^{1} \rightarrow \mathbb{R}^{n}$ be a $C^{2}$ function which is $(Q, T)$-rotating-periodic. Then $|\sigma(t)|$ is bounded on $\mathbb{R}^{1}$ with an upper bound $M_{\sigma}$. Let $\varphi(t)=(-C, \ldots,-C)^{T}$ and $\psi(t)=(C, \ldots, C)^{T}$, where $C=M_{\sigma}+1$. Then $\varphi(t)<\psi(t)$ are lower and upper solutions of (25). It is easy to 
see that $\varphi(t)$ and $\psi(t)$ satisfy the Kamke type condition (K) and condition (16) with $c=1$. Furthermore, for any $t \in \mathbb{R}^{1}, \varphi(t) \leq x \leq \psi(t)$ and $y \in \mathbb{R}^{n}$, we have

$$
\begin{aligned}
& \langle x, A(t, x) y+B(t) x-e(t)\rangle \leq M_{A}|x||y|+M_{B}|x|^{2}+n M_{e} C, \\
& |\langle y, A(t, x) y+B(t) x-e(t)\rangle| \leq h_{A}(|y|)|y|,
\end{aligned}
$$

where $M_{A}$ is an upper bound of $\|A(t, x)\|$ on $\left\{(t, x) \mid \varphi(t) \leq x \leq \psi(t), t \in \mathbb{R}^{1}\right\}, M_{B}$ and $M_{e}$ are upper bounds of $\|B(t)\|$ and $|e(t)|$ on $t \in \mathbb{R}^{1}$, respectively, $h_{A}(s)=M_{A} s+n M_{B} C+M_{e}$ satisfies (7). Hence conditions (17) and (18) in Theorem 2 hold. Then there exists a $(Q, T)$ rotating-periodic solution.

\section{Acknowledgements}

The authors would like to thank two anonymous referees whose comments and suggestions have improved the presentation of the paper. They also appreciate Prof. Yong Li for his useful suggestions. Jin Zhang wishes to express her deep gratitude to Prof. Michael Y. Li and the University of Alberta for hospitality when the final version of the work was prepared.

\section{Funding}

This work is partially supported by CSC Scholarship.

\section{Availability of data and materials}

Not applicable.

\section{Competing interests}

The authors declare that they have no competing interests.

\section{Authors' contributions}

All authors have jointly completed this work and they read and approved the final manuscript.

\section{Author details}

${ }^{1}$ School of Mathematics, Jilin University, Changchun, China. ${ }^{2}$ Center for Mathematics and Interdisciplinary Sciences, Northeast Normal University, Changchun, China.

\section{Publisher's Note}

Springer Nature remains neutral with regard to jurisdictional claims in published maps and institutional affiliations.

Received: 23 May 2019 Accepted: 21 January 2020 Published online: 30 January 2020

\section{References}

1. Andres, J., Górniewicz, L.: Topological Fixed Point Principles for Boundary Value Problems. Topological Fixed Point Theory and Its Applications, vol. 1. Kluwer, Dordrecht (2003)

2. Andres, J., Malaguti, L., Pavlačková, M.: Hartman-type conditions for multivalued Dirichlet problem in abstract spaces. In: Discrete Contin. Dyn. Syst. Dynamical Systems, Differential Equations and Applications, 10th AIMS Conf. Suppl., pp. 38-55 (2015)

3. Barkley, D., Kness, M., Tuckerman, L.S.: Spiral-wave dynamics in a simple model of excitable media: the transition from simple to compound rotation. Phys. Rev. A 42, 2489-2491 (1990)

4. Chang, X. Li, Y.: Rotating periodic solutions of second order dissipative dynamical systems. Discrete Contin. Dyn. Syst. 36, 643-652 (2016)

5. Chang, X., Li, Y.: Rotating periodic solutions for second-order dynamical systems with singularities of repulsive type Math. Methods Appl. Sci. 40, 3092-3099 (2017)

6. De Coster, C., Habets, P.: Two-Point Boundary Value Problems: Lower and Upper Solutions. Mathematics in Science and Engineering, vol. 205. Elsevier, Amsterdam (2006)

7. Dong, Y.: On solvability of three point BVPs of second order ODEs. J. Math. Anal. Appl. 296, 131-139 (2004)

8. Fabry, C., Habets, P.: The Picard boundary value problem for nonlinear second order vector differential equations. J. Differ. Equ. 42, 186-198 (1981)

9. Hofer, H., Zehnder, E.: Symplectic Invariants and Hamiltonian Dynamics. Modern Birkhäuser Classics. Birkhäuser, Basel (2011)

10. Kamke, E.: Zur theorie der systeme gewöhnlicher differentialgleichungen II. Acta Math. 58, 57-85 (1932)

11. Knobloch, H.W., Schmitt, K.: Non-linear boundary value problems for systems of differential equations. Proc. R. Soc. Edinb. A 78, 139-159 (1977)

12. Lasota, A., Yorke, J.A.: Existence of solutions of two-point boundary value problems for nonlinear systems. J. Differ Equ. 11, 509-518 (1972)

13. Liu, G., Li, Y., Yang, X.: Rotating periodic solutions for asymptotically linear second-order Hamiltonian systems with resonance at infinity. Math. Methods Appl. Sci. 40, 7139-7150 (2017) 
14. Liu, G., Li, Y., Yang, X.: Existence and multiplicity of rotating periodic solutions for resonant Hamiltonian systems. J. Differ. Equ. 265, 1324-1352 (2018)

15. Liu, G., Li, Y., Yang, X.: Infinitely many rotating periodic solutions for second-order Hamiltonian systems. J. Dyn. Control Syst. 25, 159-174 (2019)

16. Long, Y.: Index Theory for Symplectic Paths with Applications. Progress in Mathematics, vol. 207. Birkhäuser, Basel (2002)

17. Mawhin, J.: The Bernstein-Nagumo problem and two-point boundary value problems for ordinary differential equations. In: Qualitative Theory of Differential Equations, Vol. I, II, Szeged (1979)

18. Mawhin, J.: Topological Degree Methods in Nonlinear Boundary Value Problems. CBMS Regional Conference Series in Mathematics, vol. 40. Am. Math. Soc., Providence (1979)

19. Mawhin, J.: Two point boundary value problems for nonlinear second order differential equations in Hilbert spaces. Tohoku Math. J. 32, 225-233 (1980)

20. Skinner, G.S., Swinney, H.L.: Periodic to quasiperiodic transition of chemical spiral rotation. Phys. D, Nonlinear Phenom. 48, 1-16 (1991)

21. Xu, F., Yang, X., Li, Y., Liu, M.: Existence of affine-periodic solutions to Newton affine-periodic systems. J. Dyn. Control Syst. 25, 437-455 (2019)

22. Yan, P., Zhang, M.: Higher order non-resonance for differential equations with singularities. Math. Methods Appl. Sci. 26, 1067-1074 (2003)

23. Yang, X., Zhang, Y., Li, Y.: Existence of rotating-periodic solutions for nonlinear systems via upper and lower solutions. Rocky Mt. J. Math. 47, 2423-2438 (2017)

24. Zhang, Y., Yang, X., Li, Y.: Affine-periodic solutions for dissipative systems. Abstr. Appl. Anal. 2013, Art. ID 157140 (2013)

\section{Submit your manuscript to a SpringerOpen ${ }^{\circ}$ journal and benefit from:}

- Convenient online submission

- Rigorous peer review

- Open access: articles freely available online

- High visibility within the field

- Retaining the copyright to your article

Submit your next manuscript at $\gg$ springeropen.com 